\title{
COMERCIO ÉTNICO E INTEGRACIÓN SOCIAL: ANÁLISIS DEL COMERCIO POLACO EN LA CUENCA DEL RUHR
}

\author{
Ana Espinosa Seguí \\ Departamento de Geografía Humana \\ Universidad de Alicante
}

\section{RESUMEN}

El comercio étnico posee una gran representatividad espacial y social en la economía étnica. Por ese motivo, el comercio puede convertirse en una oportunidad de socialización de los empresarios inmigrantes con la sociedad y economía receptoras, sobre todo teniendo en cuenta que el colectivo de inmigrantes es uno de los más desfavorecidos en sociedades postindustriales, el autoempleo en el sector terciario se ha convertido en una de las alternativas más viables al desempleo industrial. En este artículo se ha realizado un análisis sobre los comercios regentados por empresarios polacos en la Cuenca del Ruhr alemana con la finalidad de analizar el grado de integración territorial, social y comercial que estos comercios poseen, dependiendo de su localización e interacción con la sociedad y economía de base.

Palabras clave: economía étnica, comercio étnico, integración social, enclave étnico, Cuenca del Ruhr.

\begin{abstract}
Ethnic shops have large spatial and social representativity among the entrepreneurial activities of the ethnic economy. Ethnic retail may become a good opportunity for immigrants, as long as it is inserted in a positive social and geographical atmosphere, mostly when immigrants are one the most disadvantaged groups of post-industrial economies in order to find a new job. For this reason, self-employment is considered a viable alternative against industrial unemploy-
\end{abstract}


ment. In this article, an empirical research has been carried out in the Ruhr Area among Polish grocery stores in order to analyse the grade of social, territorial and commercial integration of these shops in the society and economy of the host country.

Key words: ethnic economy: ethnic economy, ethnic shops, social integration, ethnic enclave, Ruhr Area.

\section{INTRODUCCIÓN}

El comercio y la restauración étnica son dos de las actividades pertenecientes a la economía étnica que gozan de mayor visualización en los espacios urbanos (Leung, 2007), además de estar consideradas como nichos de negocio (Light, 2007) preferentes para la mayoría de comunidades étnicas. Conforme el fenómeno migratorio de un país ha ido creciendo a lo largo de los años, el volumen de establecimientos comerciales y de restauración regentados por inmigrantes ha aumentado de forma significativa.

En un principio, estos establecimientos surgen como respuesta a la demanda de la población coétnica que intenta, en la medida de lo posible, mantener los hábitos de consumo y de estilo de vida propios de sus países (Wilpert, 2003; Galbraith et al., 2007) como parte del proyecto inmigratorio que han iniciado. De este modo, una gran parte de las actividades pertenecientes a la economía étnica tienen como punto de partida el servicio a la propia comunidad étnica y el abastecimiento de bienes y servicios procedentes de sus países de origen.

Sin embargo, con el paso del tiempo estos establecimientos comerciales y de restauración maduran y se van incrustando en la cultura del país de acogida, diluyendo la rígida relación entre demanda y oferta de los mismos y por ende, ganando consumidores para sus propios negocios (Aramburu, 2002; Wilpert, 2003; Espinosa, 2008, 2011) en parte de la población receptora y entre otros colectivos de inmigrantes.

En este proceso, el inmigrante que ha optado por el autoempleo en un negocio de su propiedad está interactuando con su colectivo coétnico, y con la sociedad de base, es decir, con otros inmigrantes y con la sociedad receptora ${ }^{1}$, ofreciendo un servicio no sólo comercial, sino también cultural y social, pero también con el territorio.

1 En esta investigación se utilizarán las expresiones economía y sociedad de base para referirse a la matriz social y económica que encuentra el empresario inmigrante en la sociedad de acogida. La sociedad de base se refiere no sólo a la población autóctona, sino también a los otros colectivos de inmigrantes, mientras que la expresión economía de base haría referencia a las actividades económicas que lleva a cabo la sociedad de acogida y otros empresarios étnicos. 
Así, su presencia física en una determinada calle o barrio va a ayudar a la configuración de un tejido comercial, y por ende, un tejido social y cultural con unas características definitorias (Aramburu, 2002; Moreras, 2007).

Por ello, de la capacidad de interaccionar con la sociedad y el espacio que un comerciante pueda llegar a poseer, dependerá en gran medida su éxito en la integración comercial y sobre todo social en la ciudad y en la sociedad de base, aglutinando este último concepto no sólo a la población del país receptor sino también al resto de comunidades inmigradas.

Esto no significa que la mayor presencia en la actualidad de espacios propios de la comunidad inmigrante en las ciudades de acogida de muchas ciudades europeas haya supuesto una mejora de la interacción de una comunidad (Fong y Ooka, 2000) con la sociedad de base.

Incluso se podría hablar de un proceso negativo en ciertas áreas de la ciudad, en las que la mayoría de la población y de la economía local tienen un origen étnico y encuentran serias dificultades para poder relacionarse e integrarse con otras áreas urbanas o comunidades sociales que no comparten aspectos de su cultura, nacionalidad o religión.

En Alemania, la economía étnica se encuentra fuertemente asentada entre el colectivo de inmigrantes, sobre todo en la mitad Occidental del país, donde los procesos inmigratorios desde finales del siglo XIX hasta la década de los años 1970 del siglo XX conformaron una sociedad multicultural con un gran número de inmigrantes temporales y permanentes que poco a poco han ido conformando sus propias redes sociales y económicas y evidentemente, sus propios espacios urbanos y de consumo (Wilpert, 2003; Hospers, 2004; Bruder y Räthke-Döppner, 2008).

En este país, las altas tasas de empleo por cuenta propia de la comunidad inmigrante, incluso superiores a las de la población autóctona, han favorecido que la economía étnica haya sido interiorizada tradicionalmente como «las formas de autoempleo de los extranjeros» (Hillmann, 2006). Sobre todo debido a la falta de recursos laborales para los inmigrantes tras la crisis industrial y el aumento significativo del desempleo entre este colectivo a partir de los años 1990 (Ehses, 2006).

Uno de los colectivos inmigrantes con más tradición histórica en Alemania y con mayor presencia en la economía étnica del país es el polaco, por lo que en esta investigación se ha realizado un estudio empírico sobre el comercio étnico polaco en la Ruhrgebiet o Cuenca del Ruhr.

Analizando el comercio polaco instalado en esta región industrial, se va a realizar una aproximación al potencial que el comercio étnico puede tener como elemento de unión e integración social del colectivo inmigrante con la sociedad y economía de base. El estudio estará condicionado por las particularidades del grupo étnico analizado, aunque puedan ser extrapoladas muchas de las caracte- 
rísticas que poseen los comerciantes polacos a otros grupos de emprendedores extranjeros.

El planteamiento teórico se basará en literatura específica sobre la economía y comercio étnicos. Así, se señalarán las principales teorías explicativas de la economía étnica, que indudablemente contienen aspectos clave como el capital social, los procesos encadenados de vacancy chain e invasión-sucesión o la caracterización de los agentes participantes del comercio étnico. Seguidamente, se pasará a analizar el caso de estudio de la Cuenca del Ruhr, laboratorio de experimentación de la validez de la hipótesis de partida, donde la investigación inductiva y cualitativa se ha llevado a cabo. Posteriormente se presentarán los resultados del estudio. Ya que este estudio tiene una base eminentemente geográfica, se valorará el papel del territorio escala municipal y regional en el grado de socialización e integración de la comunidad inmigrante dentro de la sociedad de base tanto desde un punto de vista comercial, social como cultural.

\section{EVOLUCIÓN DE LOS ESTUDIOS SOBRE LAS RELACIONES ENTRE LA ECONOMÍA ÉTNICA Y EL TERRITORIO}

Hasta el despegue de los estudios sobre economía étnica en las ciudades occidentales, las investigaciones comerciales estaban centradas en el análisis de esta actividad con una visión excesivamente economicista.

La función social que la actividad comercial posee ha estado infravalorada en ocasiones por la imposibilidad de ligar dos conceptos diversos: la distribución comercial y las prácticas sociales.

A pesar de que la crisis del modelo comercial tradicional en Europa ha traído consigo una vasta producción literaria centrada en el papel no tanto económico, sino social, que los pequeños comercios han jugado en la creación de comunidades sociales en el centro urbano y sobre todo, en barrios consolidados (Aramburu, 2002; Moreras, 2007; Espinosa, 2008), el inicio de los estudios de economía étnica marcaron un hito en la concepción de la economía étnica como una herramienta de integración laboral y social de comunidades que presentaban una clara desventaja, cuando no discriminación, en las sociedades de acogida (Fong y Ooka, 2000; Light, 2007).

En 1994, Ivan Light y Krageorgis definieron la economía étnica como aquella compuesta de «los autoempleados y empleadores, sus trabajadores familiares no asalariados, y sus empleados coétnicos». Esta definición implicaba que diversos agentes estaban implicados de manera formal o informal, directa o indirectamente en la actividad por vínculos familiares, de amistad o étnicos.

Debido a la gran diversificación y ampliación de actividades de la economía étnica Ivan Light (2007) amplió la definición unos años más tarde, reuniendo 
bajo dos categorías todas las actividades de la economía étnica: la economía de propiedad étnica, que enfatiza el mayor control del empresariado étnico y la economía étnicamente controlada, que hace referencia a la participación de la comunidad inmigrante de una empresa, a pesar de no ser el propietario y/o inversor.

\subsection{Análisis de la literatura existente sobre economía étnica}

A pesar de la tradición inmigratoria de los países de Europa Central y de Estados Unidos, la investigación sobre la economía y comercio étnicos ha tenido un desarrollo relativamente reciente, ya que fue a lo largo de las décadas de 1970 y 1980 cuando los primeros estudiosos, procedentes de Estados Unidos, publicaron sus resultados preliminares sobre el despegue de las actividades económicas de las comunidades coreanas y cubanas en su país (Wilson y Portes, 1980; Light y Bonacich, 1988; Haller, 2004).

A partir de ese momento, las investigaciones se centraron en el análisis de las principales causas por las que los inmigrantes han optado por el autoempleo en sus países de acogida (Vid. Tabla 1).

Tabla 1. Inicios de la economía étnica

\section{PRINCIPALES CAUSAS}

Respuesta cultural y económica a un mercado de trabajo en la sociedad y economía de base más fragmentado y hostil para los inmigrantes (Bonacich, 1973; Kaplan y Li, 2006; Volery, 2007; Wahlbeck, 2007; Light, 2007); Equilibrio en aquellas industrias donde las demandas del desarrollo económico se encuentran en equilibrio con los recursos informales de la población étnica (Light y Rosestein, 1995); Nichos de mercado sin cubrir (Waldinger, 1984; Volery, 2007); Trampolín para adquirir un avance económico y social en la sociedad de base (Wahlbeck, 2007); Diferencias culturales entre la sociedad inmigrada y la sociedad receptora: a mayor diferencia, mayor será la necesidad de bienes étnicos y el nicho del mercado potencial (Volery, 2007)

\section{BASE EN LA QUE SE FORJA LA ECONOMÍA ÉTNICA}

1. SOCIOCULTURAL: predisposición de algunos colectivos para el sector comercial (Kaplan y Li, 2006); preferencia del autoempleo ante la posibilidad de trabajo en sectores con nulo o escaso reconocimiento social (Hillmann, 2006; Leung, 2007), existencia de un capital social (Portes y Landolt, 1996; Woolcock, 1998; Galbraith et al., 2007;) que favorece la formación de un club de agentes étnicos (Galbraith et al., 2007); Conocimiento relacional (Staring, 2000)

2. EMPRESARIAL: Los empresarios étnicos invierten en sectores con débil necesidad de educación y de capital (Volery, 2007; Wahlbeck, 2007)

3. INSTITUCIONAL: Empleo de políticas de desarrollo del Estado del Bienestar; consideración, por parte del Estado, del trabajo como herramienta de inserción social (Wahlbeck, 2007)

4. ECONÓMICA: Desaceleración económica en Occidente (Volery, 2007); Alternativa al desempleo mayor del colectivo de inmigrantes (Wahlbeck, 2007); Oportunidades en ciertos nichos de mercado (Kaplan y Li, 2006); reestructuración local y global (Kaplan y Li, 2006)

5. TERRITORIAL: Abandono comercial de ciertos barrios por parte del empresariado de base (Arjona y Checa, 2006); La proximidad geográfica puede crear sinergias comerciales (Kaplan y Li, 2006)

Elaboración propia a partir de la bibliografía citada en el cuadro 
Al inicio, los estudios sobre economía étnica intentaron explicar si ha sido la mayor predisposición cultural que los ciudadanos de ciertos países poseen para el autoempleo, la principal causa por la que muchos ciudadanos inmigrantes han decidido montar sus propias empresas, (Suttles, 1972). Conocida como la aproximación culturalista (Arjona y Checa, 2006) o teoría cultural (Staring, 2000), el debate científico inicial y sobre todo actual, la ha considerado excesivamente determinista e insuficiente para poder explicar un fenómeno que desborda la perspectiva cultural y que interacciona de manera extraordinaria en el territorio y la sociedad de base (Fairlie y Meyer, 1996).

Se enfatizaban en exceso las características culturales de grupos sociales que se encuentran en un territorio y sociedad que coartaba, en mayor o menor medida, sus decisiones económicas y sociales y las iniciativas empresariales que quieran emprender, por el desconocimiento, incomprensión y dificultades de integración que puede presentar este colectivo.

Por ese motivo, en la llamada teoría de la desventaja (Bonacich, 1973) o teoría contextual (Staring, 2000), se incluyeron elementos tan importantes como la sociedad de base y el territorio, que interactúan con el empresario inmigrante y provocan su respuesta cultural y económica a un problema de incapacidad de integración social del inmigrante y de la sociedad de base. El contexto social y económico en el que se incorpora el empresario inmigrante es así decisivo para montar su propio negocio y salir fuera del circuito de empleo ofrecido por empresarios de la sociedad de base.

La teoría de la desventaja exacerba el papel del capital social (Vid. Tabla 2) que poseen los inmigrados en el país de acogida. Los recursos propios de la comunidad a la que pertenece el inmigrante es la principal baza con la que persistir en su idea de autonomía laboral, ya que permite que el empresario se apoye en sus propias redes sociales (familiares y amigos, tanto cercanos como conocidos) para establecer su negocio. Así, el capital social enfatiza el valor de las redes sociales, la cooperación étnica y la interacción social en la puesta en marcha de un negocio, sin negar la importancia de la independencia económica como elemento fundamental de la economía étnica.

Otra teoría, la ecológica inspirada en la Escuela de Chicago, toma como base los estudios de ecología urbana de Burguess, Wirthle y Park para explicar el nacimiento y desarrollo de la economía étnica en un determinado territorio (Arjona y Checa, 2006). El propio mecanismo urbano y económico de la ciudad genera zonas abandonadas por los empresarios locales que a su vez son ocupadas por los empresarios étnicos, mediante un proceso de invasión-sucesión. Este proceso supone también la ocupación de los nichos de mercado y de trabajo (vacancy chain) que los empresarios locales habían descuidado bien por falta de interés o de potencial económico (Moreras, 2007). 
Tabla 2. El capital social en la economía étnica

\begin{tabular}{|c|c|}
\hline $\begin{array}{l}\text { - (Portes, 1995): La capacidad de los individu } \\
\text { participación en redes o estructuras sociales más a } \\
\text { - (Woolcock, 1998): Ventajas, recursos o capacida } \\
\text { voluntad y conocimiento entre una bien definidac } \\
\text { red coétnica. } \\
\text { - (Nederveen, 2003): Los recursos colectivos que } \\
\text { empresa. }\end{array}$ & $\begin{array}{l}\text { social: } \\
\text { para ordenar recursos escasos en virtud de su } \\
\text { aplias. } \\
\text { es que surgen de la confianza, reciprocidad, buena } \\
\text { munidad/Ventajas colectivas provistas a nivel de la } \\
\text { s inmigrantes utilizan para establecer una pequeña }\end{array}$ \\
\hline La comunidad étnica ofrece: & El empresario étnico ofrece: \\
\hline $\begin{array}{l}\text { Redes intra-grupo (Arjona y Checa, 2006), que } \\
\text { ayudarán al propio colectivo más que a otros } \\
\text { empresarios externos a la comunidad. }\end{array}$ & $\begin{array}{l}\text { Cobertura en un nicho de mercado que la } \\
\text { sociedad de base no ha sabido aprovechar para } \\
\text { un colectivo de inmigrantes (Light, 2007) }\end{array}$ \\
\hline $\begin{array}{l}\text { Solidaridad étnica: los miembros ofrecen } \\
\text { confianza y lealtad intergrupal como } \\
\text { consecuencia del principio de solidaridad étnica }\end{array}$ & $\begin{array}{c}\text { Provisión de productos culinarios y otros bienes } \\
\text { culturales a los coétnicos (Waldinger, 1984; } \\
\text { Fertala, 2007) }\end{array}$ \\
\hline $\begin{array}{l}\text { Capital humano, financiero (Kaplan y Li, 2006) } \\
\text { y cultural (Volery, 2007), muy útil como ayuda } \\
\text { dentro de aquellos grupos que no poseen una } \\
\text { confianza recíproca con la sociedad de base. }\end{array}$ & $\begin{array}{l}\text { Preferencia en el empleo a sus coétnicos } \\
\text { (Wahlbeck, 2007) aunque llegue también a la } \\
\text { explotación laboral (Sanders y Nee, 1987; Arjona } \\
\text { y Checa, 2006; Kaplan y Li, 2006) }\end{array}$ \\
\hline $\begin{array}{c}\text { Un nicho potencial de consumidores para el } \\
\text { empresario que le asegurará un mercado mínimo } \\
\text { para minimizar su inversión }\end{array}$ & $\begin{array}{l}\text { La marcación de un territorio étnico (Kaplan y } \\
\qquad \mathrm{Li}, 2006 \text { ) para su comunidad }\end{array}$ \\
\hline La comunidad étnica recibe: & El empresario étnico recibe: \\
\hline $\begin{array}{l}\text { Apoyo de su comunidad, sobre todo si ésta ha } \\
\text { sido discriminada (Fong y Ooka, 2000) o alguno } \\
\text { de sus miembros no se ha socializado con la } \\
\text { sociedad de base por problemas de adaptación al } \\
\text { nuevo medio social o por acabar de llegar. }\end{array}$ & $\begin{array}{l}\text { Estabilidad y movilidad que le minimizan el } \\
\text { riesgo y el coste de su inversión empresarial } \\
\text { (Light y Bonacich, 1988) }\end{array}$ \\
\hline $\begin{array}{l}\text { Especialización en determinados servicios de su } \\
\text { propia comunidad étnica, sobre todo comercio, } \\
\text { restauración o servicios financieros (Kaplan y Li, } \\
\text { 2006). }\end{array}$ & $\begin{array}{l}\text { Información de los recursos existents y las } \\
\text { necesidades imperantes de su propia comunidad } \\
\text { étnica (Granovetter, 1973) aunque limite los } \\
\text { flujos de información desde el exterior de la } \\
\text { comunidad (Burt, 1992) }\end{array}$ \\
\hline $\begin{array}{c}\text { Proximidad: en ciertos servicios para aquellos } \\
\text { miembros de la comunidad que no poseen } \\
\text { movilidad (Kaplan y Li, 2006) y trabajo próximo } \\
\text { a su lugar de residencia. }\end{array}$ & $\begin{array}{c}\text { La conformación de un ambiente empresarial } \\
\text { proclive a la inversion étnica en un determinado } \\
\text { espacio que puede generar un espacio } \\
\text { económicamente sinérgico. }\end{array}$ \\
\hline
\end{tabular}

Elaboración propia a partir de la bibliografía citada en el cuadro 
Abierto un nuevo mercado, el empresario será capaz de generar una plusvalía al espacio abandonado por sus predecesores. Así, los procesos encadenados de vacancy chain e invasión-sucesión suponen una modificación sustancial del paisaje urbano (Arjona y Checa, 2006; Moreras, 2007) y la recomposición social del entramado empresarial de una calle, barrio o un área urbana de mayores dimensiones.

Esta teoría parte de la carencia de oportunidades y servicios para los inmigrados en las primeras fases de llegada (Light, 2007), la adaptación al país de acogida y la generación espontánea o planificada de servicios específicos para la comunidad cuando ésta se amplía y comienza a exigir servicios o bienes a los que estaba acostumbrado en su país de origen.

Sin embargo, ni la predisposición de una determinada comunidad inmigrante a desarrollar una actividad empresarial, ni las desventajas de los inmigrantes para poder establecerse laboralmente en su territorio de acogida, como tampoco la existencia de nichos de mercado carentes de actividad en la ciudad son las únicas explicaciones para que empresarios étnicos establezcan una actividad económica.

El ambiente laboral y social relativamente hostil que la sociedad de acogida ha ofrecido desde su llegada a los inmigrantes (Haller, 2004; Kaplan y Li, 2006; Volery, 2007; Wahlbeck, 2007; Light, 2007), o la combinación de ambos factores suponen una estructura externa de oportunidades difícil para los inmigrados.

A esta estructura impuesta por la sociedad de base y el territorio de acogida le podemos sumar las posibilidades propias que el inmigrado o la comunidad inmigrada poseen para poder labrarse un futuro de forma más autónoma, como la herencia cultural relacionada con la inversión empresarial y el capital social del que dispone el empresario, que nos da como resultado un ambiente más propicio para el florecimiento de los comercios étnicos que para el trabajo asalariado (Light y Rosenstein, 1995). Así se podría definir la tesis de la teoría interactiva, que añade elementos pasivos y activos al propio empresario inmigrado de suma importancia en el desarrollo de su actividad.

Robert Kloosterman et al. (1999) adaptó parte de la teoría interactiva en su adaptación al territorio europeo, ya que la consideraba demasiado economicista. En los países europeos se infravalora la política institucional y las posibilidades reales de los inmigrados para establecerse en el mercado de un determinado país.

Esta estructura de posibilidades y oportunidades para el inmigrado, conocida como incrustación social (mixed embeddness) valora no sólo el ambien- 
te económico, en este caso la estructura de la oferta comercial, o la capacidad empresarial del inmigrado, sino el régimen normativo en el que va a tener que establecerse el empresario y también la propia demanda de los productos que comercialice (Arjona y Checa, 2006).

De modo que cada país europeo, o cada grupo de países europeos, como propone Kloosterman (2000) presentará un marco normativo diverso que facilitará una mayor o menor incrustación social.

Así, países como Irlanda o Reino Unido mantendrían la mayor proximidad a la teoría interactiva coherente en el ámbito norteamericano, mientras que países como Austria, Francia, Luxemburgo y Alemania pertenecerían a la región «Rhineland» y Dinamarca y Suecia formarían parte de la región nórdica. Posteriormente, Arjona y Checa (2006) incluyeron a los países del sur como el Modelo de Europa del Sur, con un régimen normativo que presenta más obstáculos al inmigrante en la formalización de su proyecto laboral y social.

Alemania, perteneciente a la región «Rhineland», muestra problemas significativos para la inserción laboral de los inmigrados. El control estatal de los flujos migratorios y la estructura de oportunidades crea un modelo dicotómico de integración laboral, con personas dentro y fuera de los principales circuitos laborales.

\subsection{El comercio étnico: agentes participantes e inserción en el mercado comercial local}

De todas las actividades que comprenden la economía étnica, el comercio étnico posee una alta representación espacial y carga simbólica. En el acto de compra de productos étnicos intervienen diferentes elementos de un protocolo social establecido de forma espontánea entre el consumidor y el comerciante.

El uso del mismo idioma materno y la complicidad de ambos agentes por compartir una herencia cultural similar se exacerban en el acto de compra, llegando esta buena sintonía a generar estructuras sociales extremadamente complejas que benefician a todos los miembros de la comunidad. Como una actividad de la economía étnica, en la puesta en marcha de un comercio étnico intervienen múltiples factores no sólo relacionados con la presencia de un nicho de mercado sin cubrir o la predisposición del comerciante étnico en la puesta en marcha de un establecimiento comercial. Una vez instalado el comerciante en un determinado territorio, podemos definir cuatro agentes básicos que van a conformar la oferta, la demanda y la base territorial donde se establezca el comercio étnico (Vid. Tabla 3): el propio empresario étnico, el mercado, la demanda y el territorio. 
Tabla 3. Principales características de los agentes intervinientes en el comercio étnico

\begin{tabular}{|c|c|c|}
\hline \multicolumn{3}{|l|}{ Agentes del comercio étnico } \\
\hline \multicolumn{3}{|c|}{$\begin{array}{l}\text { 1. EMPRESARIO ÉTNICO: } \\
\text { - puede presentar un bagaje empresarial en su país de origen o de acogida, o haber estado en } \\
\text { contacto con el mundo empresarial por medio de su entorno más próximo } \\
\text { - la coyuntura laboral del país de acogida fuerza al empresario inmigrante a mejorar su estatus social } \\
\text { y laboral por medio del autoempleo formal o informal } \\
\text { - Apoyado por el capital social, no sólo desde un punto de vista laboral, social, cultural y económico. } \\
\text { - más flexible y orientado al servicio a los clientes, más arriesgado y motivado para trabajar duro que } \\
\text { muchos de los empresarios de la sociedad receptora } \\
\text { - empresarios comerciales independientes, contribuyen con su concepto del espacio comercial a la } \\
\text { remodelación del comercio tradicional por medio de la introducción de mayor volumen de servicios } \\
\text { a la demanda }\end{array}$} \\
\hline $\begin{array}{l}\text { 2. TERRITORIO: } \\
\text { - la estructura institucional de } \\
\text { cada país influirá decisivamente } \\
\text { en la economía étnica en } \\
\text { diversas escalas: nacional, } \\
\text { regional y local } \\
\text { - áreas desestimadas por los } \\
\text { empresarios de la sociedad de } \\
\text { acogida } \\
\text { - sinergias del empresariado } \\
\text { étnico }\end{array}$ & $\begin{array}{l}\text { 3. MERCADO: } \\
\text { - mayoritariamente de escala } \\
\text { local } \\
\text { - fuerte competencia con otros } \\
\text { formatos comerciales aunque el } \\
\text { posea características culturales } \\
\text { insustituibles para los coétnicos } \\
\text { - clima favorable donde insertar } \\
\text { los comercios étnicos. } \\
\text { - dinamismo comercial } \\
\text { provocado por los procesos de } \\
\text { invasión-sucesión }\end{array}$ & $\begin{array}{l}\text { 4. DEMANDA: } \\
\text { - coétnica en el inicio de } \\
\text { la actividad, aunque se ha } \\
\text { convertido en una demanda } \\
\text { más heterogénea una vez que el } \\
\text { comercio se haya inserto dentro } \\
\text { de la economía y sociedad de } \\
\text { base y haya madurado } \\
\text { - gran fidelidad causada por los } \\
\text { vínculos comunes existentes } \\
\text { - Aumento de transumers }\end{array}$ \\
\hline
\end{tabular}

Elaboración propia a partir de las lecturas de Kloosterman et. al, 2001; Wilpert, 2003 y Bruder y Räthke-Döppner (2008)

El territorio también se considera como un agente activo y no pasivo donde se desarrolla la actividad comercial, ya que posee una determinada estructura institucional, a diferentes escalas (nacional, regional o local) que va a modificar el tipo y el número de comercios étnicos. El mercado incluiría a otros comercios étnicos, que bien pueden ser sinérgicos entre ellos o competencia directa, y también al resto de la oferta comercial de la ciudad o área urbana.

De hecho, la localización espacial del comercio étnico ha sido uno de los aspectos más estudiados y analizados dentro de los estudios de la economía étnica (Portes, 1981; Portes y Bach, 1985; Kaplan y Li, 2006; Volery, 2007; Wahlbeck, 2007). La construcción del espacio económico étnico supone un proceso de territorialidad por parte de los inmigrados del espacio urbano, ya que los empresarios étnicos tendrán diversos grados de poder para elegir un área urbana y territorializarla.

En este proceso, el espacio no es un agente pasivo, ya que junto a los diversos agentes de la sociedad de base, de la comunidad inmigrante y de la dinámica social y/o del mercado inmobiliario de los establecimientos comerciales, influirá en la creación de sinergias empresariales de comercios étnicos. 


\section{LA CUENCA DEL RUHR: ESPACIO DE OPORTUNIDAD COMERCIAL PARA EL COLECTIVO INMIGRANTE}

La Ruhrgebiet, en adelante Cuenca del Ruhr, es una región industrial de gran tradición industrial perteneciente a la región alemana de Renania del Norte Westfalia (Vid. Figura 1).

Su desarrollo económico e industrial se remonta a mediados del siglo XIX, cuando tuvo un fortísimo crecimiento poblacional (Hospers, 2004) que se materializó en la creación de una de las regiones más densamente pobladas y urbanizadas de toda Europa. Constituida por 53 ciudades, muchas de ellas formando conurbaciones en dirección este-oeste, la Cuenca del Ruhr posee en la actualidad más de 5,2 millones de habitantes ${ }^{2}$, con una densidad de 230 habitantes $/ \mathrm{km}^{2}$.

Figura 1. La Ruhrgebiet o Cuenca del Ruhr

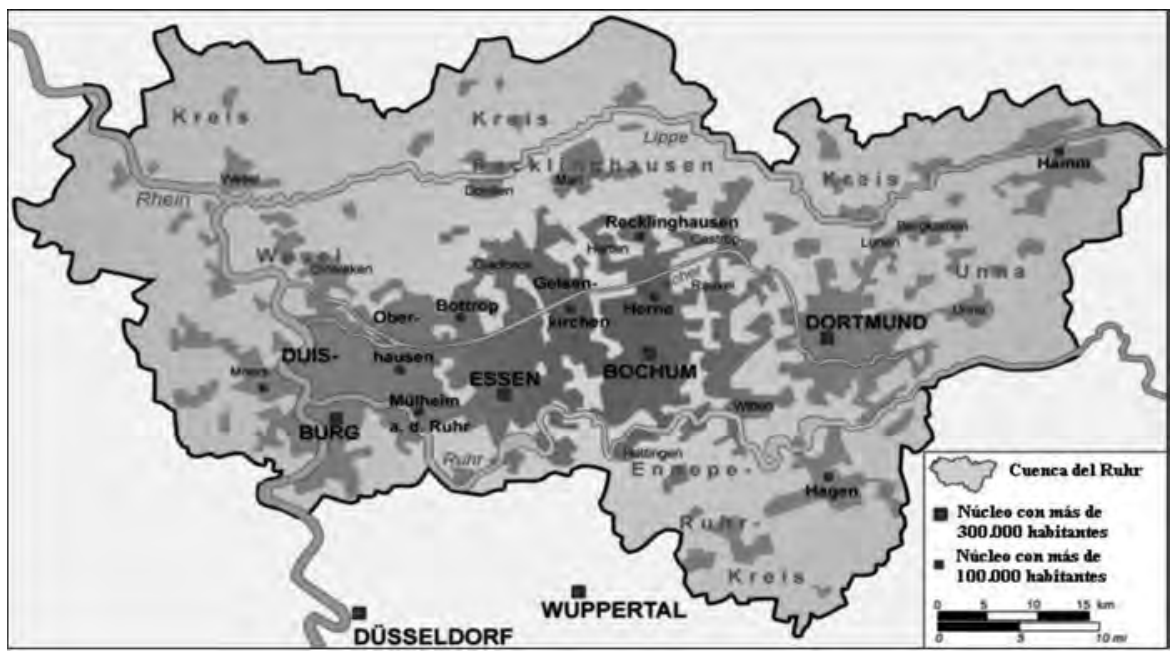

Fuente: http://www.metropoleruhr.de. Modificado por la autora

La Cuenca del Ruhr se ha distinguido tradicionalmente por la composición e identidad de su población (Hospers, 2004). La combinación de trabajadores industriales locales o inmigrantes de la propia Alemania, una creciente clase media y población inmigrante o descendientes de inmigrantes ha creado una mezcla cultural y social mucho más rica que en otras regiones alemanas, donde no fue tan necesaria la llamada de población inmigrante de otros países europeos.

2 Fuente: http://www.metropoleruhr.de/metropole-ruhr/daten-fakten/bevoelkerung.html 
Gert-Jan Hospers distingue dos principales oleadas de inmigración en la Cuenca del Ruhr, comenzando con el 1890-1918, en el que muchos polacos recibieron permisos de trabajo para desplazarse a Alemania y trabajar en la industria pesada de la región (Wilpert, 2003; Floeting y Reimann, 2004; Ferrero, 2005; Leung, 2007). La llegada y asentamiento de la comunidad polaca en la Cuenca del Ruhr no estuvo exenta de preocupación por parte de los alemanes, que intentaron limitar las asociaciones culturales y sindicatos de trabajadores polacos con el fin de limitar la territorialización de la comunidad en la región.

Posteriormente, ya en la década de 1960, la oleada de inmigración provino de los países mediterráneos del sur, básicamente Turquía, Grecia, Italia, los países de la antigua Yugoslavia y España. 39302298

La fuerte crisis industrial que comenzó entrada la década de los años 1970 supuso un fuerte revés para la situación laboral de los trabajadores de la región, tanto que la desocupación ha llegado a establecerse como el principal problema de la Cuenca del Ruhr (Hospers, 2004).

La crisis industrial aceleró el retorno de muchos de estos inmigrantes, como los españoles, aunque gran parte de los inmigrantes menos cualificados se quedaron en la región desplazando sus trabajos al sector terciario (Wilpert, 2003), en claro aumento como consecuencia de la depresión del sector secundario. Así, en el año 2009, 615.000 ciudadanos de la Cuenca del Ruhr son inmigrantes o descendientes de inmigrantes, destacando los turcos, ex-yugoslavos, polacos e italianos como las comunidades más numerosas (Vid. Figura 2).

Figura 2. Residentes extranjeros en la Cuenca del Ruhr. Año 2009

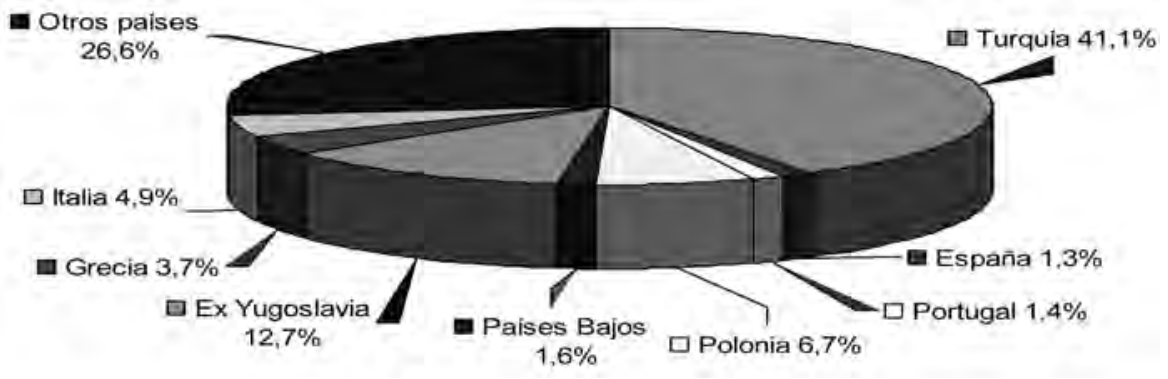

Fuente: http://www.metropoleruhr.de/ (Acceso 01/03/2011)

Tras la crisis de la década de los años ochenta, en el año 1988 el paro superó el 15\% en la región, una cifra muy elevada para Alemania que lentamente se ha conseguido bajar hasta el 10,9\% del año $2010^{3}$. La mayor proporción de desem-

\footnotetext{
3 Fuente: http://www.metropoleruhr.de/
} 
pleados son extranjeros, llegando en el año 2009 a superar el 25\% (Vid. Figura 3), aunque también es destacable el paro estructural instaurado en la región de trabajadores alemanes poco cualificados que perdieron sus empleos con la crisis industrial.

Una parte de esta tasa de paro ha conseguido rebajarse gracias al autoempleo al que se acogieron tanto la población inmigrante como alemana, considerándolo una medida para paliar los problemas de desempleo de la región (Fischer, 2001; Goch, 2002).

Los servicios en general, y sobre todo el sector comercial han sido algunas de las actividades económicas que más han crecido en los últimos años. Parte de la población extranjera se ha autoempleado a través del comercio y servicios, aunque también es cierto que la gran dificultad de buena parte de los extranjeros residentes en la región por escapar del desempleo ha generado una infraclase social (Goch, 2002), formada mayoritariamente por extranjeros con una baja cualificación laboral y escasos conocimientos de la lengua alemana (Fischer, 2001).

En este contexto de inadaptación laboral, los inmigrantes polacos no han sido una de las comunidades más perjudicadas (Fischer, 2001; Fertala, 2006), como ha sucedido con los inmigrantes procedentes de Turquía, los más numerosos de la Cuenca del Ruhr y de Alemania, o los inmigrantes asiáticos.

En primer lugar, desde finales del siglo XIX Alemania ha sido el destino de un gran número de trabajadores, Gastarbeiter, muchos de ellos polacos (Ferrero, 2005; Leung, 2007), por lo que se creó una comunidad numerosa que consiguió lentamente integrarse aún manteniendo sus tradiciones.

Figura 3. Población activa desempleada en los años 2009 y 2010 en la Cuenca del Ruhr

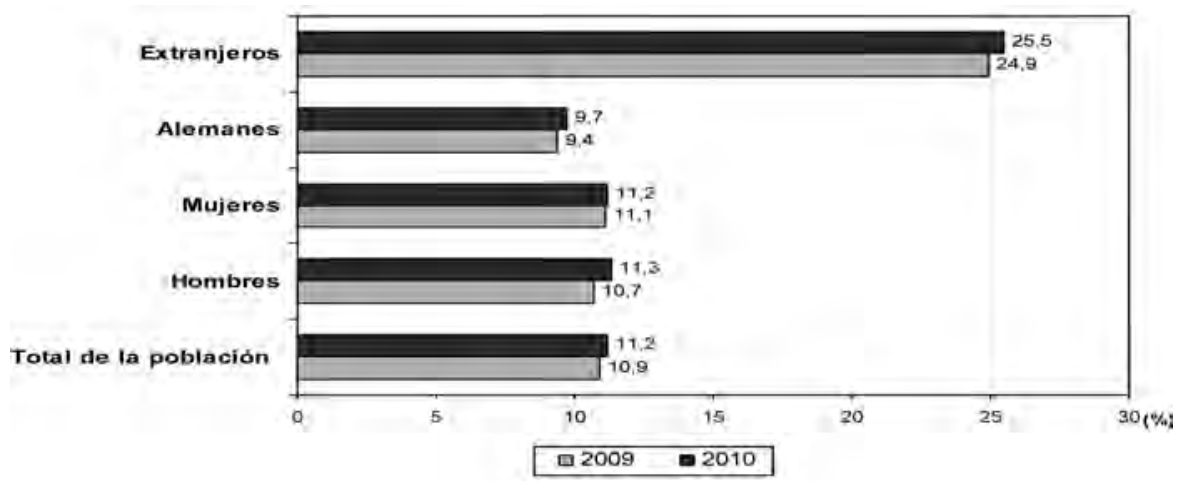

Fuente: http://www.metropoleruhr.de/ (Acceso 01/03/2011) 
Este asentamiento paulatino de los inmigrantes polacos durante más de un siglo en Alemania, fundamentalmente en su mitad Occidental, provocó una consiguiente mezcla cultural y social por lo que hoy son comunes los apellidos polacos en parte de la población alemana y los descendientes, ya alemanes, de antiguos inmigrados. Otro elemento que es necesario tener en cuenta es el retorno de los alemanes étnicos, o de sus descendientes, provenientes de los antiguos territorios alemanes, hoy parte de Polonia tras el Tratado de Potsdam.

Según el Bundesamt für Migration und Flüchtlinge, en 2006 la contribución de la comunidad polaca a la economía étnica del país se encuentra en segundo puesto (16\%), sólo superada por los empresarios turcos (23\%), aunque con una participación más baja de los primeros en el sector comercial.

Además, según apuntan Bruder y Räthke-Döppner (2008), esta fuerte iniciativa empresarial de los inmigrados polacos en Alemania puede explicarse en parte por las facilidades de movimiento que ofrece el gobierno alemán a los trabajadores que registren un negocio.

Esta actividad empresarial gestionada por inmigrados polacos ha estado, contrariamente a lo que sucede con la comunidad turca o vietnamita, centrada en el sector de la construcción, limitando a sólo 7\% el número de empresas comerciales gestionadas por polacos en Alemania.

Bruder y Räthke-Döppner proponen la tesis de que en sectores como la distribución comercial, la mayor reticencia de algunas comunidades se relaciona con los años de permanencia prevista en el país de acogida. De este modo, la cercanía con Polonia condiciona el sector de actividad donde el inmigrante quiere autoemplearse durante el período de permanencia en el país, limitando la puesta en marcha de aquellas actividades que dependan del entorno empresarial y social donde se localizan. Y así, es fácil encontrar entre los comercios en activo de inmigrantes polacos, comercios puramente étnicos, nacidos para cubrir la demanda de los co-étnicos, y también aunque en medidas variables, de la sociedad de base.

\section{CUESTIONES METODOLÓGICAS}

La mayor profusión de investigaciones sobre la economía étnica con un enfoque espacial y/o económico plantea interrogantes acerca de la capacidad de integración que el autoempleo o el empleo de terceros en comercios étnicos pueda tener en los empresarios inmigrantes (Fong y Ooka, 2000).

La labor socializadora del comercio presupone una mayor implicación de estos comerciantes en la zona en la que han instalado su establecimiento comercial y mayor contacto con la sociedad de base. Por ese motivo, la hipótesis de partida de este estudio plantea que la actividad comercial puede poseer mayor capacidad para integrar a los comerciantes étnicos que otras actividades laborales sin tanto contacto social. 
Y por ello, el objetivo es analizar el papel que el comercio étnico ha tenido en la integración social de sus propietarios en la sociedad de acogida.

Integración social entendida como la estabilidad de las relaciones entre partes dentro de un sistema social o el proceso por el que se unen, mejoran o se mantienen unidos entre sí elementos diversos formando una nueva estructura, según definición de la Fundación Europea para la Mejora de las Condiciones de Vida y de Trabajo.

En este sentido, y tomando como eje principal de la investigación la posible capacidad de integración social del comercio étnico, se establecieron tres cuestiones de investigación:

- ies el comercio étnico una herramienta eficaz de integración social para el colectivo de inmigrantes étnicos?

- ¿qué estrategias han seguido los comerciantes étnicos para abrirse (en un posible caso positivo de integración) a la sociedad de base?

- iel posible proceso de integración social del empresario inmigrante se ha realizado a causa del comercio que regenta?

Para este estudio y en una fase preliminar, se seleccionaron sólo las actividades empresariales comerciales de la economía de propiedad étnica, ya que se buscaba interactuar con comerciantes polacos autoempleados, propietarios y gestores de sus establecimientos. El surtido debía estar claramente orientado a los bienes de consumo tipificados como étnicos, que están mejor representados por los productos de alimentación polacos y debía existir una identificación externa visible del comercio étnico, excluyendo los restaurantes o comercios regentados por empresarios polacos de bienes o servicios sin una clara orientación étnica.

En este sentido, las corrientes inmigratorias iniciadas ya en el siglo XIX y la llegada reciente del colectivo polaco a la Cuenca del Ruhr ha ayudado a establecer un gran número de comercios y empresas de servicios regentados por polacos, o descendientes ya alemanes de inmigrantes polacos, caracterizadas como no étnicas.

Con el fin de analizar en profundidad las opiniones y experiencias personales del empresariado polaco, se realizó un análisis cualitativo, que facilitó la labor de conocer de primera mano la información requerida, «al permitir el despliegue de las diferencias que existen entre cada uno de los sujetos«(Sánchez, 1995). Así, se descartó la cuantificación de información estadística de la muestra, ya que no habría podido reflejar con total claridad y profundidad las experiencias vividas desde que se inició el proyecto migratorio de cada uno de los entrevistados.

De este modo, se eligió la entrevista semiestructurada como base para la recopilación de información cualitativa de los entrevistados. A pesar de la libertad 
que se les dejó a los entrevistados para poder estructurar su ideas y vivencias, en su entrevista se utilizó un cuestionario con preguntas abiertas para centrar la discusión en la integración social de los comerciantes étnicos. Así, las preguntas se centraban en la opinión que tenían sobre las oportunidades de negocio y de integración de sus comercios, las características de sus clientes, los posibles problemas que han padecido, la importancia de la localización en la ciudad y las estrategias sociales y económicas seguidas desde el inicio de la actividad comercial

Se realizaron un total de 11 entrevistas a empresarios polacos en activo con establecimientos comerciales en la Cuenca del Ruhr, de un total de 14 empresas contabilizadas por la autora. Las entrevistas se realizaron personalmente, con un traductor polaco y alemán, durante los meses de marzo a mayo del año 2010, con una duración que osciló entre 35 y 70 minutos.

Al ser un estudio cualitativo, era conveniente contar con un universo de pequeño volumen pero con el que se pudiera trabajar con mayor profundidad. Se planteó no aumentar el número de entrevistas con otros colectivos étnicos diversos para no distorsionar la información ofrecida por un colectivo determinado. Además, en la elección del grupo de empresarios a analizar se tuvo en cuenta el grado de adaptación al país de acogida. Así, y en general, los comerciantes polacos no habían sufrido graves conflictos con la sociedad de base en el proceso de asentamiento e integración en el país.

Ello no significa que los resultados obtenidos puedan ser extrapolados por completo a otros empresarios étnicos, que pueden haber sufrido mayores problemas en su llegada y adaptación al país de acogida.

Debido al cambio de local comercial y a la elevada mortalidad de los comercios étnicos polacos, se tuvo que confiar en la información proporcionada por los propios comerciantes, la información recogida en internet y también, en la información ofrecida por los distribuidores de productos étnicos polacos de la región de Westfalia Renania del Norte.

El método de investigación utilizado ha sido el inductivo, que basado en la observación empírica de una determinada área geográfica ayuda, en parte, y teniendo en cuenta las limitaciones que el territorio y universo de estudio plantean, a la construcción de una teoría general.

\section{RESULTADOS DE LA INVESTIGACIÓN}

Tras realizar la investigación cualitativa en los comercios étnicos regentados por empresarios polacos en la Cuenca del Ruhr, se realizó una codificación de los códigos más citados por los entrevistados (Vid. Tabla 4), en los que se pudo ver cómo muchos comerciantes consideraban sus establecimientos como herramientas para potenciar su identidad nacional. 
Tabla 4. Codificación de la información cualitativa de los entrevistados

\begin{tabular}{|c|c|}
\hline CÓDIGOS ANALÍTICOS & DESCRIPCIÓN \\
\hline Identidad & $\begin{array}{l}\text { Los entrevistados reconocían que el comercio les ha ayudado a } \\
\text { reforzar su identidad en Alemania, aunque también ha supuesto el } \\
\text { efecto contrario en otros casos, en los que el comerciante busca la } \\
\text { ruptura con la identidad de su país de origen. }\end{array}$ \\
\hline Comunicación & $\begin{array}{l}\text { La comunicación con personas que son nativos en el mismo idioma } \\
\text { del entrevistado se ha valorado como un elemento importante e } \\
\text { insustituible a la hora de realizar operaciones empresariales o de } \\
\text { entablar lazos de amistad. }\end{array}$ \\
\hline Rechazo social & $\begin{array}{l}\text { Aunque ninguno de los entrevistados ha sufrido situaciones graves } \\
\text { de rechazo o xenofobia, sí que se ha dado cuenta de que muchos } \\
\text { alemanes los considerarán siempre como extranjeros. }\end{array}$ \\
\hline Patriotismo & $\begin{array}{l}\text { Muy relacionado con el código «identidad», los comerciantes } \\
\text { declaraban que su comercio era una extensión de su país, por lo que } \\
\text { generaba en ellos y sus clientes una exacerbación del patriotismo. }\end{array}$ \\
\hline Integración social & $\begin{array}{l}\text { Además de ampliar su círculo de conocidos y clientes de origen } \\
\text { polaco, gran parte de los entrevistados declaró que es fundamental } \\
\text { un trabajo al público para poder integrarse mejor en el lugar de } \\
\text { residencia y trabajo. }\end{array}$ \\
\hline
\end{tabular}

Elaboración propia tras la realización del trabajo de campo

Del mismo modo, esa identidad nacional más clara y abierta a la sociedad de base les ayudaba a integrarse mejor en el territorio, tanto con sus comercios vecinos como con los clientes. Una vez establecidos los principales códigos de información proporcionados por los entrevistados, se va a proceder a explicar los resultados obtenidos atendiendo a las cuestiones de investigación planteadas en el inicio de la investigación.

\subsection{El comercio étnico como herramienta de integración social}

Uno de los elementos más importantes que citaban los entrevistados se relacionaba con la ganancia de mayor y más clara identidad cultural respecto a la sociedad de base, ya que el establecimiento de un comercio étnico reforzaba su procedencia. Tal y como un empresario polaco de un comercio de Dortmund expresaba: "con esta tienda quizás no me he integrado mejor, pero me ha ayudado a encontrar un lugar en esta sociedad. Antes, tenía que justificarme y explicar de dónde soy. Trabajaba como taxista y siempre me decían ¿De dónde vienes? (...) Aquí saben que soy polaco y que vendo productos de Polonia. Aquí yo soy yo mismo». 
Otro comerciante afirmaba que el comercio étnico que regentaba había afianzado su situación social, ayudándole a explicar a la sociedad de base su situación personal: "Para mi integración creo que el comercio sí que me ha ayudado. Yo tengo la nacionalidad alemana, pero soy un «aussiedler ${ }^{4}$. Nací en Polonia y vine a Alemania con mi familia de pequeño, y por eso recibí el pasaporte alemán. Yo soy consciente de que no me aceptan al 100\% como alemán [...] Por eso, para mí el comercio ha sido muy bueno. No me tengo que justificar siempre de mi situación, explicar que mis abuelos eran alemanes pero que siempre hemos hablado polaco en casa. La mayor parte de mis clientes vinieron a Alemania como yo, hace unos veinte años o antes, como mano de obra extranjera para poder trabajar aquí. Ellos están contentos de poder hablar polaco y yo también».

Esta posibilidad que brindan los comerciantes étnicos polacos de poder comunicarse con sus clientes, vecinos u otros comerciantes en dos idiomas (polaco y alemán), también se considera un aspecto clave en el proceso de integración social: "la gente viene aquí para hablar conmigo en polaco, y muchas veces me preguntan las novedades de Polonia. Si he oído una noticia importante (...) La gente está contenta de poder venir a la tienda y de hablar de sus cosas. Aquí se sienten un poco mejor».

Además, según Nikolinka Fertala (2007), en el ámbito empresarial los polacos prefieren tratar con otros polacos por la barrera idiomática que muchos poseen y también por la confianza mutua que se crea entre ellos.

Es evidente que el volumen principal de clientes que reciben los comerciantes entrevistados son connacionales, por lo que estos espacios de intercambio comercial, se han convertido en espacios sociales de comunicación y asesoramiento, como así narraba uno de los entrevistados: "No es solamente un punto para comprar. Algunas veces me llaman para preguntarme si conozco un médico que habla polaco, o cualquier otro servicio... se fían de mí».

Sin embargo, también es posible que en estos comercios con clara identidad nacional se hayan dado casos de problemas en la comunicación entre empresarios y clientes, debido a la incierta identidad social de algunos inmigrados, como el que relataba uno de los entrevistados: «Tengo un cliente polaco que habla muy mal el alemán, pero no hablaría nunca conmigo en polaco porque se sentiría ofendido. Al principio, nos enfadábamos mucho porque yo no podía entender la razón por la que él actuaba así.»

4 Bajo la denominación «Aussiedler» se contempla la población alemana que tras un período de residencia prolongado fuera de Alemania, normalmente en países de Europa del Este vuelve a vivir en Alemania. También se consideran aussiedler los nacidos en el extranjero de alemanes, que pueden tener las dos nacionalidades. 
La situación que relataba este comerciante podría estar relacionada con la histórica relación entre alemanes y polacos en la Cuenca del Ruhr, ya que desde los inicios de su inmigración, los polacos tuvieron que luchar para conseguir su integración sociocultural, percibiendo las dificultades inherentes que su nacionalidad y su lengua provocaban en la región.

Por otra parte, si los comerciantes étnicos se abren a la sociedad de base, pueden atraer también a clientes del propio país, que bien ya conocen los productos o bien tienen interés por la cultura polaca. En esta inmersión cultural de los clientes en el comercio étnico, la labor del comerciante es fundamental. Por una parte, es decisivo que el comerciante quiera abrir su área de mercado no sólo a sus clientes y amigos connacionales, sino también a la sociedad de base.

En este sentido, un requisito indispensable es que el comercio y el comerciante estén preparados para atender a los nuevos clientes, tanto desde la perspectiva lingüística y social como den una atención al cliente personalizada. Un entrevistado que había procurado desde la apertura de su establecimiento abrirse a la sociedad de base se extrañaba de que su esfuerzo de integración y apertura de su comercio al mayor número posible de consumidores no fuera algo natural para muchos clientes: «Algunas veces vienen los alemanes y piensan que no sé hablar en alemán. Y no lo entiendo... porque si abro un comercio en Alemania se supone que sé hablar la lengua del país.»

El trato a los clientes nuevos que llegan al comercio sin conocer una parte o la totalidad de los productos ofertados es algo fundamental, y es una labor necesaria por parte del empresario étnico. Al igual que en cualquier comercio especializado, también es necesaria una mayor implicación del comerciante para aconsejar al cliente, explicar el producto, su uso o preparación: «cuando llega gente de Alemania, y piden información de los productos de Polonia, yo puedo explicárselo mejor que en un gran comercio. Por eso yo también me encuentro bien de estar aquí».

Sin embargo, sólo una comerciante consideró que el comercio no la había ayudado a establecerse mejor en su nueva sociedad, ya que prefería desvincularse de su cultura una vez establecida en Alemania, a pesar de que regentaba un comercio especializado en productos de alimentación polacos: «Aquí en Alemania he comenzado una nueva vida. No quiero sentirme siempre una de allá (Polonia) Esto es algo que siempre he tenido muy claro [...] Aquí me he integrado, me he casado y no quiero tener nada que ver con la historia polaca. Simplemente, no me siento ni he sido nunca una patriota».

\subsection{Estrategias de integración positivas del comerciante étnico}

Al igual que el comercio étnico se había consolidado como una herramienta eficaz para mejorar la identidad de sus comerciantes, es posible afirmar que este mismo sentimiento de identidad se reforzaba con la exaltación del patriotismo y viceversa. 
Dado que los empresarios étnicos entrevistados asumían que es difícil que vayan a ser tratados como nativos en diversos aspectos, por sus dificultades o limitaciones en la comunicación verbal o escrita, por su diversa cultura, planteamiento de su proyecto migratorio o por su estilo de vida, los entrevistados han optado por reforzar su identidad polaca, convirtiéndolo en su medio de vida.

Para ello, se inclinaban por cambiar la imagen de Polonia que se tiene en Alemania, por mostrar su riqueza cultural y gastronómica y por exaltar los aspectos más positivos de su país. Un entrevistado comentaba que «quiero cambiar un poco la imagen de Polonia, que no sólo tiene prados y caballos, sino también ciudades y productos de alimentación de buena calidad y buen sabor».

Esta demostración de la cultura y tradiciones polacas se debe hacer para incentivar la compra de consumidores no connacionales y para poder abrirse a la sociedad de base, por medio de diversas estrategias, que supondrán al comerciante una mayor apertura de su comercio, y consecuentemente una mayor posibilidad de interacción y futura integración social. Así, otro comerciante afirmaba que $475 \%$ de los clientes son polacos, $10 \%$ o $15 \%$ son alemanes que se han ido de vacaciones alguna vez a Polonia, el resto es un poco de todo... Hay veces que vienen los griegos o italianos de la Cuenca del Ruhr, porque les gustan los sabores fuertes, pero los turcos ya no vienen... Tengo también carne como pollo, pero quizás ellos (inmigrantes turcos) no lo saben...»

Un comercio había atraído a un gran número de consumidores rusos y bálticos por poder hablar en ruso con ellos y por las similares tradiciones culinarias de estos países con Polonia: «sin saberlo muy bien, instalé el comercio en un barrio con muchos residentes rusos y lituanos. Nunca pensé que vendrían a mi comercio a comprar cosas polacas, pero un poco por casualidad comencé a hablar con unos vecinos y nos hicimos amigos (...) También es verdad que otros vecinos del barrio, también rusos, me quitaron hasta tres veces el cartel de la tienda de la carretera porque decían que era ilegal. Pedí permiso al ayuntamiento y no había nada de malo en ello, pero con ellos nunca he podido tener una buena relación. Me ven, me saludan, saben quién soy pero no entran a comprar... Al menos no hablan mal de mí al resto del barrio».

También es posible que si el comerciante no es consciente de que su comercio es percibido y catalogado por los consumidores como étnico, no debería seguir ninguna estrategia especial para atraer a la sociedad de base aunque es muy probable que plantee su establecimiento comercial como un espacio abierto a toda los posibles consumidores: «no tengo una estrategia de mercado. Me gustaría poder tener a toda la gente como consumidores potenciales. No quiero quedarme sólo con el grupo de polacos". 
En definitiva, las estrategias comerciales que han llevado a cabo los comerciantes polacos en la Cuenca del Ruhr se han centrado no tanto en adaptar su surtido y productos a las exigencias de los consumidores, sino en incrementar el contacto social con comunidades externas a la polaca e intentar profundizar más en un servicio al cliente más cercano.

\subsection{Localización espacial del establecimiento comercial y su papel en la integración del comerciante étnico.}

En el caso de estudio de la Cuenca del Ruhr, los comercios étnicos regentados por empresarios polacos no se encontraban en zonas comerciales con una alta densidad de comercios étnicos, conocidos también como enclaves étnicos (Walbeck, 2007), sino dispersos por zonas comerciales secundarias, actuando así de tienda tradicional de barrio. Ya que la población polaca residente en la Cuenca del Ruhr no presenta una concentración residencial muy acusada en determinados áreas urbanas o rurales, tal y como sí lo hacen otras comunidades tales como la turca (Floeting y Reimann, 2004), tampoco el comercio étnico polaco presenta ninguna concentración espacial.

A pesar de ello, estos comercios étnicos se han conformado como espacios de encuentro, por lo que los emigrados los buscan con relativa frecuencia para poder realizar sus compras: «Yo no fui el primero en abrir este comercio, pero sé que lo abrieron porque había muchos polacos que viven en Dortmund. Al final, la gente llega a todas partes si quiere... Si yo estuviera en un centro comercial, si me lo pudiera permitir, no tendría esta cercanía a los clientes (...) También podría cambiar el sitio, pagar menos y tener una mejor situación, pero la gente sabe que estoy aquí y no quiero moverme por eso».

Mientras que la gran parte de comercios deben buscar un área de atracción comercial primaria y secundaria que aglutine a los residentes más próximos, en el caso del comercio étnico, la singularidad del surtido del establecimiento y la simbiosis que se crea entre empresario y consumidores facilita la captación y mantenimiento de los clientes: "La tienda es un punto de encuentro. El último jueves, en Semana Santa, había tanta gente que venía a comprar un pequeño pedazo de Polonia para tener en casa... La tienda estaba abarrotada de polacos, pero también los alemanes venían a por los productos típicos polacos".

En este sentido, uno de los ejemplos más significativos de la relación de simbiosis entre comerciantes étnicos y sus consumidores en este estudio fue el de una pequeña tienda de alimentación de productos polacos situada a menos de 30 metros de la Parroquia Santa Ana en la ciudad de Dortmund, una parroquia católica que realiza todos los servicios litúrgicos en polaco. La propietaria trabajaba durante los días lectivos en otro empleo, pero abría la tienda exclusivamente cuando los fieles acudían el fin de semana. 


\section{CONCLUSIONES}

La realización de este estudio empírico de base inductiva en los comercios étnicos polacos de la Cuenca del Ruhr ha supuesto corroborar la hipótesis que enfatiza la importante labor socializadora de la actividad comercial regentada por personas inmigrantes, recién llegadas o ya asentadas en el país de acogida. A pesar de que el volumen de comercios étnicos es siempre muy inferior al de comercios regentados por empresarios autóctonos, éstos tienen una mayor simbolismo y valor cultural para los emigrados de una determinada nacionalidad o cultura, ya que se convierten en espacios de socialización, encuentro y/o asesoramiento en diversos temas claves para los recién llegados como el laboral, inmobiliario, social o educacional.

En este análisis se ha observado una integración social de los comerciantes polacos muy o suficientemente positiva con el grupo de coétnicos pero también con la sociedad de base. Sin embargo, no hay que olvidar que existen comunidades con más dificultades a la hora de integrarse y abrir su comercio a otros colectivos de consumidores, por lo que la componente cultural condiciona sobremanera el grado de integración que puedan tener los comerciantes étnicos con su entorno. En algunas comunidades étnicas, la excesiva dependencia que el comerciante tiene del capital social llega a provocar un funcionamiento ineficiente del establecimiento y el aislamiento respecto a la sociedad de base.

Por ese motivo es fundamental la apertura paulatina del establecimiento a la sociedad de base. Así, en la puesta en marcha del comercio, el comerciante se ayuda de su red social o de los conocimientos que posee del capital social para establecerse en su área comercial. La búsqueda del local comercial estará más o menos influida por el capital social del que disponga, y también por los procesos de vacancy chain e invasión-sucesión. Una vez que el comercio adquiera más madurez, es previsible una reducción del uso del capital social, ya que es probable que el comerciante haya aprendido mejor la lengua del país de acogida o haya establecido redes comerciales ajenas a su capital social. Si es así, es muy probable que el comerciante amplíe su integración social en la sociedad y territorio de base, adaptándose a su nueva situación, en la que no sólo compran sus propios connacionales sino también consumidores pertenecientes a la sociedad de base. De este modo, los empresarios étnicos son capaces de mantener los vínculos con su comunidad étnica al tiempo que se abren a la sociedad de base.

En todo este proceso de apertura o mantenimiento de la estructura inicial de agentes implicados en el comercio étnico, el territorio juega un papel fundamental, ya que puede llegar a suponer un agente socializador para el comerciante o por el contrario, un agente evasor que impida la socialización del empresario étnico. 
El comercio étnico puede convertirse en un espacio bisagra de consumo y socialización que acerca la cultura y costumbres a los inmigrados de una determinada cultura o nacionalidad, al tiempo que se localizan en regiones o países que, por norma general, ofrecen mejores oportunidades económicas para los inmigrados que en sus países de origen.

En definitiva, los comercios étnicos pueden llegar a considerarse embajadores de su cultura y país, ayudando a la propia integración social entre sus promotores y la sociedad de base.

\section{AGRADECIMIENTOS}

Esta investigación se realizó gracias a la beca obtenida del Programa propio para el fomento de las relaciones internacionales del año 2010 de la Universidad de Alicante. Gracias a la gran profesionalidad y amabilidad de los comerciantes polacos entrevistados en la Cuenca del Ruhr ha sido posible obtener información fidedigna, completa y muy bien argumentada sobre el comercio polaco en la zona de estudio. Mención especial merece Stefan Wieczorek, que fue mi soporte lingüístico y traductor, sin el que este estudio no habría podido realizarse.

\section{BIBLIOGRAFÍA}

Aramburu, M. (2002): «Los comercios de inmigrantes extranjeros en Barcelona y la recomposición del inmigrante como categoría social». Scripta Nova: revista electrónica de Geografía y Ciencias Sociales, nº 108.

Arjona, A. y CheCA, J. C. (2006): «Economía étnica: teorías, conceptos y nuevos avances». Revista Internacional de Sociología, vol. LXIV, no 5, 117-143 pp.

BONACICH, E. (1973): «A theory of ethnic antagonism: the split labor market». American Sociological Review, vol. 38, 583-594 pp.

BRUder, J. y RäTHKe-DöPPNER, S. (2008): Ethnic minority self-employment in Germany: geographical distribution and determinants of regional variation, Working paper $n^{\circ} 100$, Witschafts und Szialwissenschaftliche Fakultäat, ThünenReihe Angewandter Volkswirtschaftstheorie, 1-30 pp.

BURT, R. (1992):»Structural holes: the social structure of competition, Harvard University Press.

EHSEs, B. (2006): The Ruhrgebiet: facts and figures. Essen, Regionalverband Ruhr, $121 \mathrm{pp}$.

EspinosA, A. (2008): «Comercio, centros históricos e inmigración». En Valero Escandell (ed.). La inmigración en los centros históricos, publicaciones de la Universidad de Alicante, 119-132 pp. 
Espinosa, A. (2011): Serie tipologías comerciales. Capitulo III: el comercio étnico, publicado en Open Course Ware como material docente audiovisual de la asignatura «Geografía del Comercio y los Servicios» de la Universidad de Alicante. Accesible en: http://hdl.handle.net/10045/16765

Fairlie, R. y Meyer, B. (1996): «Ethnic and racial self-employment differences and possible explanations». Journal of Human Resources, $\mathrm{n}^{\circ} 31$, 292-328 pp.

FerRero, R. (2005): Nuevos socios, nuevas fronteras: los procesos migratorios desde Europa Central y Oriental, Serie Migraciones, nº 5, CIDOB ediciones, Barcelona.

Fertala, N. (2007): «Immigrants entrepreneurs in Hamburg». En Leo Paul Dana (ed.). Handbook of research on ethnic minority entrepreneurship, Edward Elgar Publishing Limited, 511-533 pp.

Fischer, I. (2001): «Ethnische Ökonomie als Potenzial zur Stabilisierung benachteiligter Stadtteile? «, disponible en: http://www.raumplanung.tu-dortmund.de/irpud/fileadmin/irpud/content/SPZ/Dateien/IvFischer_Diplomarbeit.pdf

Floeting, H. y Reimann, B. (2004): Ethnische Ökonomie: Integrationsfaktor und Integrationsmaßstab. Deutsches Institut für Urbanistik, Berlin, 123 pp.

Fong, E. y OOKA, E. (2000): «The social consequences of participating in the ethnic economy». The International migration review, vol. 36, $\mathrm{n}^{\circ} 1,125-146 \mathrm{pp}$.

Galbraith, C.; Rodríguez, C. y Stiles, C. (2007):»Ethnic economies, social capital and the economic theory of the clubs». En Leo Paul Dana (ed.). Handbook of research on ethnic minority entrepreneurship, Edward Elgar Publishing Limited, 16-29 pp.

Goch, S. (2002): «Betterment without Airs: Social, Cultural, and Political Consequences of De-industrialization in the Ruhr». Internationaal Instituut voor Sociale Geschiedenis, no 47, 87-111 pp.

Granovetter, M. (1973): «The strength of weak ties». American Journal Sociology, vol. 78, n 6, 1360-1380 pp.

Haller, W. (2004), «Immigrant entrepreneurship in comparative perspective: rates, human capital profiles and implications of immigrant self-employment in advanced Industrialized societies», publicado en la Luxembourg Income Study Conference, June, Bourglinster, Luxemburg.

Hillmann, F. (2006): «Gendered landscapes of ethnic economies: Turkish entrepreneurs in Berlin». En Kaplan, David y Li, Wei (ed.). Landscapes of the ethnic economy, Rowman \& Littlefield publishers, Inc. Lanham, 97112 pp. 
Hospers, G. J. (2004): Regional economic change in Europe: a neo-Schumpeterian vision, Lit Verlag, Munster, Germany.

Jones, T. y Ram, M. (1998): Ethnic Minorities in Business, Small Business Research Trust, Londres.

Kaplan, D. y Li, W. (2006): «Introduction: the places of ethnic economies». En Kaplan, David y Li, Wei (ed.). Landscapes of the ethnic economy, Rowman \& Littlefield publishers, Inc, Lanham, 1-16 pp.

Kloosterman, R., Van Der Leun, J. y Rath, J. (1999): «Mixed embededdeness, (in)formal economic activities and inmigrant businesses in the Netherlands». International Journal of Urban and Regional Research, $\mathrm{n}^{\circ}$ 23, 252-266 pp.

Kloosterman, R. (2000): «Immigrant entrepreneurship and the institutional context: a theoretical exploration». En J. Rath (ed.). Immigrant Businesses: the economic, political and social environment, 90-106 pp.

Kloosterman, R., Van Der Leun, J. y Rath, J. (2001): «Immigrant entrepreneurs in advanced economies, mixed embeddedness further explored». Journal of Ethnic and Migration Studies, $\mathrm{n}^{\circ} 27,189-201 \mathrm{pp}$.

Leung, M. (2007): «Migrant entrepreneurship in Germany». En Leo Paul Dana (ed.). Handbook of research on ethnic minority entrepreneurship, Edward Elgar Publishing Limited, 669-680 pp.

Light, I. y BonaCich, E. (1988): Immigrant entrepreneurs. Korean in Los Angeles, 1965-1982, publicado por Berkeley Univeristy of California Press.

Light, I. y Karageorgis, S. (1994): «The ethnic economy», pp. 647-671. En Smelser, Neil y Swedberg, Richard (eds.). Handbook of Economic Sociology, Russell Sage Foundation, Nueva York, capítulo 26.

Light, I. y Rosestein, C. (1995): Race. Ethnicity and entrepreneurship in urban American, Hawthorne, Aldine de Gruyter, 255 pp.

Light, I. (2007): «Economías étnicas». En Empresariado étnico en España, CIDOB, Barcelona, 41-68 pp.

Moreras, J. (2007): «Iniciativas comerciales inmigrantes en un contexto urbano en transformación: el caso de Ciutat Vella (Barcelona)». En Empresariado étnico en España, CIDOB, Barcelona, 129-154 pp.

Nederveen, J. (2003): «Social Capital and Migration: Beyond Ethnic Economies». Ethnicities vol. 3, no 1, 29-58 pp.

Pagliarin, S. (2012): Empresariado étnico y formación de enclaves comerciales: el papel de las redes sociales en el caso de la calle de Sant Pau en Barcelona, http://www.ub.edu/geocrit/b3w-962.htm

Portes, A. (1981): «Modes of incorporation and theories of labour immigration». En Kritz, Keeley y Tomasi (eds.). Global Trends in Migration, Centre for Migration Studies de New York,. 279-97 pp. 
Portes, A. y BACH, R. L. (1985): Latin Journey: Cuban and Mexican Immigrants in the United States, University of California Press, Berkeley, 387 pp.

Portes, A. y Sensebrenner, J. (1993): «Embeddedness and migration: notes in the social determinants of economic action». The American Journal of Sociology, $n^{\circ}$ 98, 1320-1350 pp.

Portes, A. (1995): «Economic sociology and the sociology of immigration: a conceptual overview», en Alejandro Portes (editor): The Economic Sociology of Immigration: Essays on Networks, Ethnicity and Entrepreneurship, Russell Sage Foundation.

POWER y ElLison. (1995): «Interracial contact and black racial attitudes: the contact hypothesis and selectivity Bias». Social Forces, $\mathrm{n}^{\circ} 74,205-226$ pp.

Sánchez, M. E. (1995): «Historias de vida de inmigrantes». Didáctica, $n^{\circ} 7,271$ 278 pp, Servicio de Publicaciones UCM, Madrid.

SANDERS, J. y NeE, V. (1987): «Limits of ethnic solidarity in the enclave economy». American Sociological Review, n 52, 745-767 pp.

StARING, R. (2000): «Flows of people: globalization, migration and transnational communities» in The ends of Globalisation: bringing society back, ed. Don Kalb, Marco Van der Land, Richard Staring, Bart Van Steenbergen y Nico Wilterdink, Lanham, Rowman and Littlefield, 203-216 pp.

Suttles, G. (1972): The social construction of communities, University of Chicago Press.

VolerY, T. (2007): »Ethnic entrepreneurship: a theoretical framework». En Leo Paul Dana (ed.). Handbook of research on ethnic minority entrepreneurship, Edward Elgar Publishing Limited, 30-41 pp.

WaldingeR, R. (1984): «Immigrant enterprise in the New York garment industry». Social Problems, n 32, 60-71 pp.

WahlBeck, O. (2007): «Work in the kebab economy: a study of the ethnic economy of turkish immigrants in Finland». Ethnicities, vol. 7, no 4, 543-563 pp.

WILPERT, C. (2003): «Germany: from workers to entrepreneurs» en Immigrant entrepreneurs: venturing abroad in the age of globalization. En Robert Kloosterman y Jan Rath (ed.), Berg Publishers, capítulo 12.

Wilson, K. y Portes, A. (1980): «Immigrant enclaves: An Analysis of the Labour Market Experiences of Cubans in Miami». American Journal of Sociology, vol. 5, no 78, 295-319 pp.

Woolcock, M. (1998): «Social capital and economic development: toward a theoretical synthesis and policy framework». Theory and Society, vol. 27, 151-208 pp. 